\title{
Awards season is upon us...
}

\author{
The 2009 Nobel Prize for Physiology or Medicine goes to telomerase researchers, the Lasker Award to nuclear \\ reprogramming pioneers, and crystallographers are awarded the Chemistry Nobel once again.
}

T he trio who received the 2006 Albert Lasker Award for Basic Medical Research, Elizabeth Blackburn, Carol Greider and Jack Szostak, have now been honored with the 2009 Nobel Prize in Physiology or Medicine for the discovery of "how chromosomes are protected by telomeres and the enzyme telomerase." The "telomerase tale' is well known - the discovery that the protection of chromosome ends by telomere DNA is evolutionarily conserved, and the isolation of enzymatic activity resulting in the synthesis of new telomeric DNA. (See a Commentary by the three awardees (Nat. Med. 12, 1133-1138, 2006) and an Essay (Nat. Struct. Mol. Biol. 13, 1036-1038, 2006) by Vicki Lundblad, who was a postdoc with Szostak, recollecting her first-hand experience of the unfolding of events.)

What started out as the unraveling of a basic scientific problemunderstanding the molecular basis of chromosome ends and how they are replicated (also known as the 'end replication problem')resulted in the discovery of a medically relevant enzyme, telomerase. It soon became apparent that many cancer cell lines have abnormal telomeres and that cancer cells often have increased telomerase activity, observations that have resulted in efforts to develop therapeutic approaches targeting telomerase. A number of inherited diseases, such as dyskeratosis congenita, are known to be caused by telomerase defects, and understanding the molecular basis of these disorders will help researchers devise future therapeutics. Telomere shortening is also an important factor in the aging process, but its role in aging is complex, and research in this area is ongoing.

Given that receiving the Albert Lasker Basic Medical Research Award can be a 'sign of things to come', this year's awardees, John Gurdon and Shinya Yamanaka- "for discoveries concerning nuclear reprogramming"- are people to watch. The conceptual breakthrough that spearheaded this field came from Gurdon's studies as a $\mathrm{PhD}$ student in the late 1950s. By injecting a nucleus from an adult Xenopus laevis cell into an enucleated egg, he showed that the resulting zygote could give rise to an apparently normal adult frog, overturning the dogma that specialized cells have undergone permanent nuclear changes that cannot be reset. Four decades later, Yamanaka compiled a list of 24 genes that had been implicated in maintaining mouse embryonic stem cells, reasoning that they might be good candidates for converting fully differentiated cells into multipotent cells. He whittled these down to four genes that were able to convert mouse fibroblasts into stem cells. When injected into immunodeficient mice, the induced pluripotent stem (iPS) cells developed into various types of tissues. This technical breakthrough was subsequently used to generate human iPS cells including, most recently, the first patient-specific iPS cells. The ultimate goal is to use patient-specific iPS cells to model human disease, assess drug effectiveness and toxicity, and create tissue that might repair damage caused by injury or disease.

This year's Nobel Prize in Chemistry has been awarded to Venki Ramakrishnan, Thomas Steitz and Ada Yonath "for studies of the structure and function of the ribosome." Given that complex problems-especially ones as complex as the unraveling of the ribosome structure—often require crucial input from many players, the Nobel committee undoubtedly had to make difficult choices. In some people's minds, Harry Noller-who was the first to suggest the "possibility that $23 \mathrm{~S}$ ribosomal RNA participates in the peptidyl transferase function" and who also made significant contributions to the ribosome structure-would have been a deserving winner.

However, this should not take away from the vital contributions of the awardees. Yonath's work throughout the 1980 s resulted in a recipe for generating robust and well-diffracting ribosome crystals. This opened the way for low-, intermediate- and, eventually, high-resolution structures of the large subunit of bacterial ribosome from Steitz's group (Science 289, 905-920, 2000) and of the small subunit from Ramakrishnan's and Yonath's groups (Nature 407, 327-339, 2000; Cell $102,615-623,2000$ ). Since then the three groups, as well as other teams, have further refined the structures to understand in atomic detail how ribosomes translate genetic information into proteins. For instance, structural insight into the small subunit has been instrumental for understanding how mRNA codons are read during translation, whereas the structure of the large subunit has helped researchers dissect how ribosomes catalyze peptide bond formation. The crystal structures have also been used to show how different antibiotics bind to the ribosome, knowledge that is expected to lead to the development of sorely needed new antibiotics.

Following the 1962 Nobel Prize, awarded to James Watson, Francis Crick and Maurice Wilkins for their atomic model of DNA, and the 2006 Prize, in which Roger Kornberg was honored for determining the structure of RNA polymerase, this year's prize for work on the ribosome completes the central dogma (DNA $\rightarrow$ RNA $\rightarrow$ protein). It is also interesting to note that once again (for the third time in 7 years) the Nobel Prize in Chemistry goes to crystallographers, highlighting the significance of structural biology in chemistry. Finally, it is remarkable that the three prestigious awards concern basic scientific problems, the understanding of which, in each case, has considerable medical significance - once again stressing the importance of funding basic research.

To celebrate this year's Nobel Prizes in Medicine or Physiology and in Chemistry, we have compiled a Web Focus on telomeres and ribosomes containing recent NSMB papers in these fields (http://www.nature.com/ $\mathrm{nsmb} /$ focus/telomeres/index.html). We hope you enjoy it. 